Y. C. Minh and E. F. van Dishoeck, eds.

\title{
Masers as Kinematic Signposts in Star Formation Regions
}

\author{
Ray P. Norris \\ CSIRO Australia Telescope National Facility, PO Box 76, Epping, NSW \\ 1710, Australia
}

\begin{abstract}
Masers have been well studied as indicators of star formation regions for over three decades. Their small size, high brightness, and narrow velocity width mean that we can measure their position and velocity with enormous accuracy, and so they stand out as high-precision signposts amidst the swirling gas that they sample. Nevertheless, in most cases the complexity of their kinematics has defied attempts to use them to unravel the processes of star formation. However, the last two or three years have seen a resurgence of interest in these masers because of exciting new evidence that, in some cases, they are tracing with high precision the kinematics of material in circumstellar disks around massive stars. The very existence of circumstellar disks around these massive stars is puzzling, and yet the maser results have now been confirmed by other data at radio and infrared wavelengths. In this paper I will review the current status of high-resolution maser observations, discuss some of the puzzles that are now confronting us, and speculate on where our current tentative steps may lead us.
\end{abstract}

\section{Introduction}

Over thirty years ago, Weaver et al. (1965) discovered $\mathrm{OH}$ masers associated with HII regions in our galaxy. It quickly became clear that these masers were powerful tools for studying the kinematics of high-mass star formation. Their small size and high brightness meant that their position could be measured easily to a high precision (a few milliarcsec) using radio interferometers. Their narrow linewidths (usually less than $1 \mathrm{~km} \mathrm{~s}^{-1}$ ) meant that their velocity could also be measured easily and accurately with these same interferometers. The combination of these two measurements meant that they stood out as signposts in the interstellar medium, accurately sampling the velocity field as a function of position.

One potential drawback to their use as kinematic indicators is that the masers do not occur uniformly in space. The nature of the maser process means that it is inherently very non-linear, and so various subtle effects determine whether or not a maser is visible in a particular location. However, these effects are unlikely to influence the velocity or position of the maser, and so their use as highly accurate kinematic signposts remains valid for those regions which are sampled by the masers. In addition, the strong Zeeman splitting of the $\mathrm{OH}$ lines means that the $\mathrm{OH}$ masers, as a bonus, also tell us the magnetic 


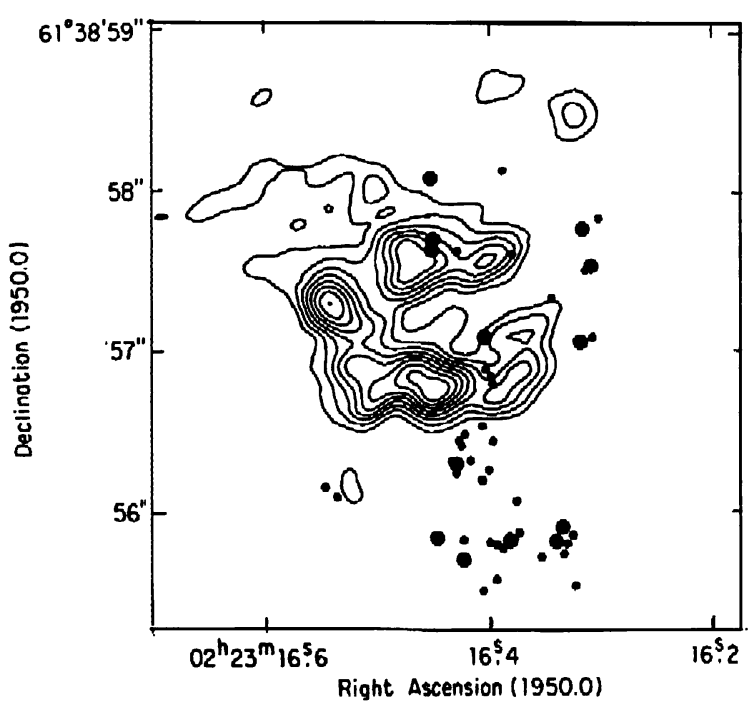

Figure 1. The $\mathrm{OH}$ masers in $\mathrm{W} 3(\mathrm{OH})$, superimposed on a radio continuum image, taken from Norris et al. (1982). Each maser is shown as a dot, and the position and velocity of each of these can be measured routinely to accuracies of a few milliarcsec and about 0.1 $\mathrm{km} \mathrm{s}^{-1}$ respectively.

field to an accuracy of a few percent. Furthermore, in principle, other detailed measurements of the lines can tell us something about the density, pressure, temperature, and abundances of the maser regions. However, to deduce these other physical parameters, it is essential to understand something of the maser mechanism itself.

Since the early work on $\mathrm{OH}$ masers, maser emission from several molecules (e.g. $\mathrm{OH}$, water, $\mathrm{SiO}$, formaldehyde, methanol) has been discovered and studied in a range of environments. However, even now, in only a few cases (such as the $\mathrm{OH}$ masers in comets and around late-type stars) do we have any real understanding of the inversion mechanism that must be present to cause the maser action. In most other cases we have little more than plausibility arguments for what actually pumps these masers, and so we are unable to use the masers to probe the physical conditions (density, temperature, etc.) in the maser gas.

Despite this embarrassing ignorance, these masers have been widely used as kinematic signposts. This is because knowledge of the maser mechanism is not actually necessary if the maser is to be used only as a signpost of kinematics. From this point on in this paper, I will ignore the internal workings of the masers, and will instead just use them as tools to probe the kinematics of the molecular gas. 


\section{Broken Promises}

In the seventies and eighties, it appeared that the detailed and accurate kinematic information available from masers might be a key tool for unraveling the problems of how stars and planets formed. As a result, many high-mass star formation regions were carefully imaged in several maser species.

A typical example is shown in Fig. 1, which shows one of the several highprecision maser images made of the ultra-compact HII (UC HII) region W3 $(\mathrm{OH})$. Despite the large amount of detailed information available from this result, no simple picture emerged. There was even a debate on whether the masers were in accreting (Reid et al. 1980) or ambient (Norris et al. 1981) gas. This confused picture was not clarified until a study of recombination lines by Welch \& Marr (1987), but even now is still a topic of debate (Kawamura \& Masson 1998). Rather than the masers revealing an underlying simple structure, it appeared that the masers were sampling an unexpected level of complexity. Instead of the naively-expected shell of warm gas sandwiched between a shock front and an ionisation front, the gas was swirling around in complex motions more reminiscent of the Earth's weather patterns than of any recognisable star formation model. In the face of this complexity, it appeared that masers might not, after all, hold the key to understanding star formation, and so the number of publications in the field declined.

\section{Why Methanol Masers Are Making New Promises}

In 1987, Batrla et al. discovered an exceptionally strong methanol maser at 12.2 $\mathrm{GHz}$ in a number of star formation regions. It was quickly found that these were extremely common (Norris et al. 1987) in massive star formation regions. Indeed they were just as common as the $\mathrm{OH}$ masers, and it was something of an embarrassment to the radio-astronomy community that these strong masers had lain undiscovered for so long.

Early interferometer observations (Norris et al. 1988) gave tantalising suggestions that perhaps some of the masers were in lines, rather than being buried in the complex structures seen in $\mathrm{OH}$ and water masers. Interest in these masers heightened in some quarters as it was realised that perhaps these masers did show relatively simple structures which were capable of being interpreted in terms of star formation models.

Interest became even more intense when Menten (1991) discovered an even stronger methanol maser transition at $6.7 \mathrm{GHz}$. Radio synthesis images quickly showed that these two new maser transitions were coincident to within a few milliarcsec (Norris et al. 1993). The same observations showed that the strong $6.7 \mathrm{GHz}$ masers were just as common as the other $\mathrm{OH}$ and methanol masers, and that some fraction of the $6.7 \mathrm{GHz}$ masers were aligned along lines. The masers that lay in lines also tended to have velocity gradients along them, as shown in Fig. 2, suggesting that they may represent edge-on circumstellar disks (Norris et al. 1998). 

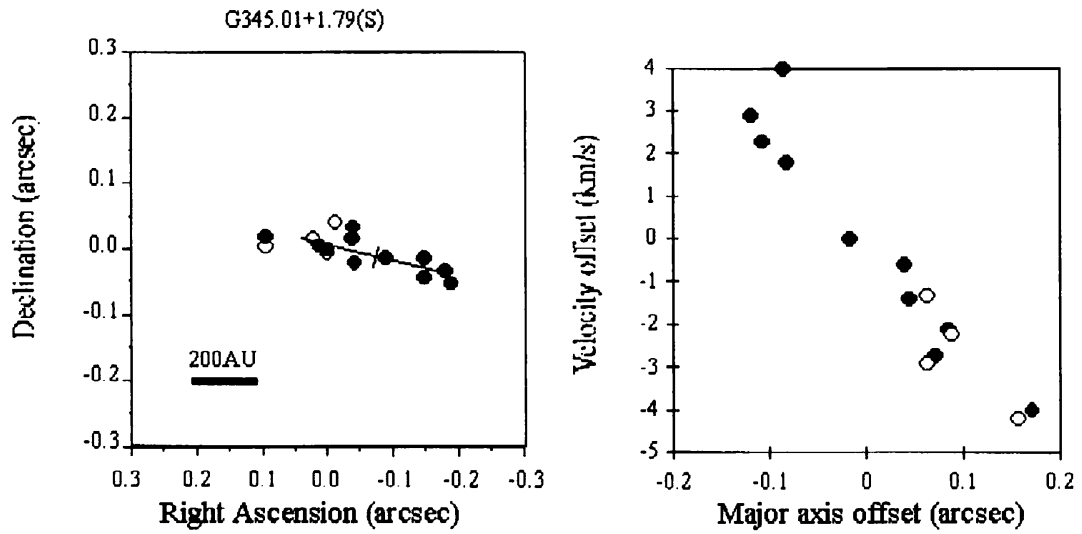

Figure 2. The line of methanol masers in G345.01+1.79(S), taken from Norris et al. (1998). Each maser is shown as a circle: open circles represent $12 \mathrm{GHz}$ masers, and filled circles represent $6.7 \mathrm{GHz}$ masers. The left-hand diagram shows the image of the masers on the sky, and the right hand diagram is a plot of position against velocity. The cross in the left hand diagram represents the position angle and origin of the fitted major axis on to which the positions have been projected to obtain the position offsets shown in the right hand diagram, the axis origin of which is arbitrary.

\section{Masers without UC HII Regions}

Most of the searches for methanol masers (e.g. McLeod \& Gaylard 1992; Caswell et al. 1995a,b) started with catalogues of known $\mathrm{OH}$ or water masers, or other indicators of star formation. Walsh et al. (1997) extended this by searching for sources selected by IRAS criteria for massive star formation. Ellingsen et al. (1996a) extended it in a different way by making an unbiased search of a section of the Galactic plane, in which a part of the plane was systematically scanned without regard to any pre-existing catalogue. In both of these surveys, methanol maser sources were discovered which did not correspond to previously known star formation indicators. In particular, it was found by Phillips et al. (1998) that about half of the methanol maser sources do not have any identifiable UC HII region in sensitive radio continuum images.

It should be noted that, whilst the lack of an optical or infrared identification could be attributed to extinction by dust, there is no similar extinction mechanism which could hide the UC HII regions at radio wavelengths. Furthermore, the sensitivity of the radio observations was such that any OB star surrounded by a Strömgren sphere would have been easily detectable.

Two mechanisms have been suggested for this. Phillips et al. suggested that perhaps some of these masers were associated with lower-mass stars that were unable to ionise a UC HII region. On the other hand, Walsh et al. suggested that perhaps these masers represented an early stage of formation of an $\mathrm{OB}$ star in which there had not yet been time to form a UC HII region. This latter 
explanation seemed unlikely in traditional models of the formation of UC HII regions, since the UC HII region forms in a time that is very short compared to its lifetime, so that the number of these sources without UC HII regions should be much less than the number with UC HII regions. On the other hand, it is possible that, for an OB star forming in a dusty medium, much of the ultraviolet radiation may be absorbed by dust, thus prolonging the growth phase of the UC HII region. It is also possible that collisions of the ionised gas with dust particles could quench the UC HII region itself. At the time of writing, this issue is unresolved.

It should also be noted that in some of the surveys, there remains a small number of methanol maser sources which are not accompanied by any signs whatsoever of star formation. It is possible that there is still a type of unidentified object, other than star formation, which is associated with methanol maser emission.

\section{Circumstellar Disks around High-Mass Stars}

The suggestion that these lines might indicate edge-on disks was initially viewed with some scepticism. This was partly because, although circumstellar disks were known to be common in low-mass star formation, the standard models of star formation suggested that such disks should not be able to survive in highmass stars. Instead, it was suggested that these lines might represent shock fronts, or tightly-collimated low-velocity jets.

Three developments seemed to point the way towards adoption of the circumstellar disk model. First, Hollenbach et al. (1994) developed a model in which an optically-thick disk around a high-mass star would provide sufficient self-shielding that the disk might have a lifetime comparable to that of the UC HII region in which it was embedded. Furthermore, ablation of the disk material would help replenish the ionised material in the UC HII region, thus extending the life of the UC HII region, and solving the dilemma that there were more UC HII regions than would be expected from previous models of their formation and evolution.

Second, Ellingsen et al. (1996b) showed that in the best-studied case, G339. 88-1.25, the line of masers neatly bisected the UC HII region, in complete agreement with the Hollenbach et al. and Norris et al. models.

Finally, Stecklum et al. (1998), using infrared imaging, appeared to confirm the hypothesis by discovering an edge-on dust disk coincident with the line of masers in this same source. At present, it appears that, at least in this one source, G339.88, there is now strong evidence that the masers are confined to an edge-on disk. Other examples are also being found elsewhere, such as the discovery of a methanol disk in the source NGC7538 by Minier et al. (1998).

An important piece of evidence for these circumstellar disks is that the derived Keplerian masses are consistent with the expected mass of the central star. Unfortunately, it is difficult to measure the Keplerian mass from a disk of test particles, as the mass depends on the cube of the overall extent of the particles, which tends to be very uncertain. In particular, the derived mass should be regarded as a lower limit rather than a measured value, because the disks will in general extend further out than the masers. Nevertheless, the derived masses 


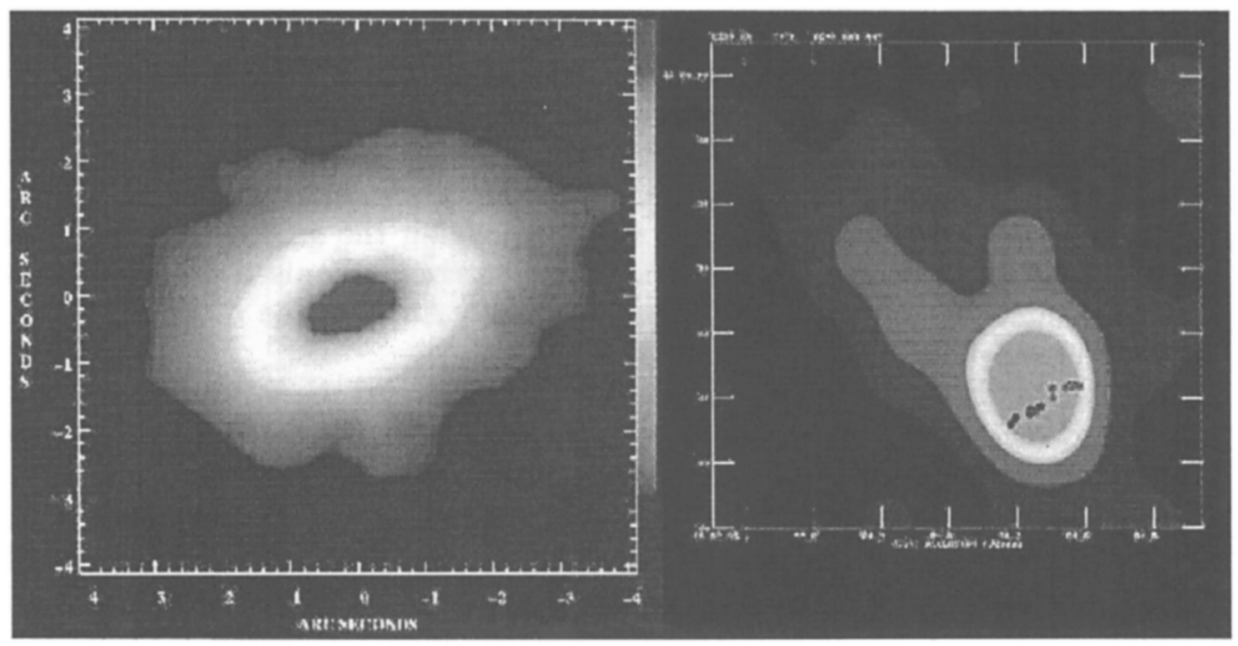

Figure 3. The edge-on disk in G339.88. The right-hand image (Ellingsen et al. 1996b) shows the masers with a monotonic velocity gradient along them (indicated by dots) superimposed on the compact HII region. Each tickmark is $1^{\prime \prime}$. The left-hand image (Stecklum et al. 1998) shows the same object at 10 micron, showing a dust disk coincident with the masers

for those maser sources with well-defined disks, shown in Table 1, are consistent with the disks being in Keplerian orbit around a high-mass star. Now that the overall geometry of the masers in well-studied sources such as G339.88 has been established, it is important to try to disentangle the large-scale features from the turbulence within the disk that is undoubtedly present. It should be possible to use the precise kinematic information to get a far more detailed rotation curve of the circumstellar disk. One technique that is available to us to obtain this detailed information is to measure the proper motion and acceleration of the masers in these sources, and thus build up a full three-dimensional characterisation of their kinematics. A number of such proper motion programs are under way, and proper motions for some sources are expected to be measured in the next year or two. Similarly, acceleration of the maser features in the spectra, over the several-year baseline since these masers were first detected, should soon be detectable.

\section{The Shape of the UC HII Region}

The UC HII region in G339.88, shown in Fig. 3, is of the class described as "cometary" by Wood \& Churchwell (1989). Such cometary UC HII regions account for about $20 \%$ of all UC HII regions. The cause of the characteristic shape has been attributed both to the motion of the UC HII region through a molecular cloud, and to a buoyancy effect, or "champagne flow", caused by 
Table 1. Derived Keplerian masses.

\begin{tabular}{llc}
\hline Source & $\begin{array}{l}\text { Diameter } \\
(\mathrm{AU})\end{array}$ & $\begin{array}{c}\text { Min. Keplerian } \\
\text { Mass }\left(\mathrm{M}_{\odot}\right)\end{array}$ \\
\hline G305.21+0.21 & 1600 & 1 \\
G309.92+0.48 & 4300 & 10 \\
G318.95-0.20 & 1000 & 4 \\
G336.42-0.26 & 2600 & 14 \\
G345+1.79(S) & 670 & 4 \\
G351.42+0.64 & 3400,1200 & 9,2 \\
\hline
\end{tabular}

the density gradient within the molecular cloud. Here I suggest a third possible cause.

It is noticeable that the cometary extension of the UC HII region in G339.88 is perpendicular to the maser disk. Whilst it is entirely possible that this is due to a chance alignment, it may also indicate that the disk itself is responsible for a buoyancy effect, and the champagne flow is directed towards the pole of the disk, rather than by a larger-scale density gradient within the molecular cloud. Based on this sample of one object, this suggestion can only be classed as a speculation at present. However, if the same alignment is found in other methanol maser sources associated with cometary UC HII regions, then it will be a strong indication that these are driven by the local density gradient of which the disk itself forms a part.

\section{Conclusion}

Interstellar masers appear to have declined in perceived significance in recent years, largely because a great deal of excellent work on them has not reaped significant benefits in our progress towards understanding the process of star formation. The methanol masers appear to be reversing this trend, and for the first time we seem to be able to relate maser structures to structures observed at other wavelengths. For those $30 \%$ of methanol maser sources which have simple spatial structures, the high precision with which they tell us the kinematics of the gas cloud in which they are embedded, means that we are able to use them as powerful probes of star formation processes. The combination of accurate synthesis observations of methanol masers, coupled with high-resolution millimetre images of other molecular gas in these objects, together with data at infrared and other wavelengths, may in the next few years finally give us a detailed picture of the kinematics of the material that forms stars and planets.

\section{References}

Batrla, W., Matthews, H.E., Menten, K.M., \& Walmsley, C.M. 1987, Nature, 326,49 
Caswell, J.L, Vaile, R.A, Ellingsen, S.P, Whiteoak, J.B., \& Norris, R.P. 1995a, MNRAS, 272, 96

Caswell, J.L., Vaile, R.A., Ellingsen, S.P., \& Norris, R.P. 1995b, MNRAS, 274, 1126

Ellingsen, S.P., Norris, R.P., \& McCulloch, P.M. 1996b, MNRAS, 279, 101

Ellingsen, S.P., Von Bibra, M.L., McCulloch, P.M., Deshpande, A.A., Norris, R.P., \& Phillips, C.J. 1996a, MNRAS, 280, 378

Hollenbach, D., Johnstone, D., Lizano, S., \& Shu, F. 1994, ApJ, 428, 654

Kawamura, J.H. \& Masson, C.R. 1998, ApJ, 509, 270

McLeod, G.C. \& Gaylard, M.J. 1992, MNRAS, 256, 519

Menten, K.M. 1991, ApJ, 380, L75

Minier, V., Booth, R. S., \& Conway, J. E. 1998, A\&A, 336, L5

Norris, R.P. \& Booth, R.S. 1981, MNRAS, 195, 213

Norris, R.P., Booth, R.S., \& Diamond, P.J. 1982, MNRAS, 201, 209

Norris, R.P., Byleveld, S.E., Diamond, P.J., Ellingsen, S.P., Kesteven, M.J., McCulloch, P.M., Reynolds, J.E., Tzioumis, A.K., Takahashi, Y., Troup, E.R., \& Wellington, K.J. 1998, ApJ, 508, 275

Norris, R.P., Caswell, J.L., Gardner, F.F., \& Wellington, K.J. 1987, ApJ, 321, L159

Norris, R.P., McCutcheon, W.H., Caswell, J.L., Wellington, K.J., Reynolds, J.E, Peng, R.S., \& Kesteven, M.J. 1988, Nature, 335, 149

Norris, R.P., Whiteoak, J.B., Caswell, J.L., Wieringa, M.H., \& Gough, R.G. 1993, ApJ, 412, 222

Phillips, C.J., Norris, R.P., Ellingsen, S.P., \& McCulloch, P.M. 1998, MNRAS, 300,1131

Reid, M.J., Haschick, A.D., Burke, B.F., Moran, J.M., Johnston, K.J., \& Swenson, G.W. 1980, ApJ, 239, 89

Stecklum, B., Henning, Th., Feldt, M., Hayward, T.L., Hoare, M.G., Hofner, P., Richter, S. 1998, AJ, 115, 767

Walsh, A.J., Hyland, A.R., Robinson, G., \& Burton, M.G. 1997, MNRAS, 291, 261

Weaver, H.F., Williams, D.R.W., Dieter, N.H., \& Lum, W.T. 1965, Nature, 208, 29

Welch, W.J. \& Marr, J. 1987, ApJ, 317, L21

Wood, D.O.S. \& Churchwell, E. 1989, ApJS, 69, 831

\section{Discussion}

D. Johnstone: Comment. If there are disks around high-mass stars then they will be evaporated and contribute to the appearance of the HII region as mentioned in my talk. Question. Are there any sources besides MWC349 where hydrogen recombination masers are observed and which yield a disk signature?

R.P. Norris: I totally agree with your comment. Perhaps this contributes to the appearance of cometary UC HII regions. I am not aware of any other $\mathrm{H}$ recombination masers.

T. Velusamy: Could you comment on the radial distribution of the masers in the disk? 
R.P. Norris: At the moment, our data do not tell us very much about this, because there are too many free parameters. For example, the sources with a monotonic velocity gradient may well represent a ring at a particular radius, and perhaps in those cases the total extent of the masers tells us the diameter of the ring. But an alternative solution is that the ring is much larger, and we are only seeing a small region of masers on the nearside of the ring.

M. Burton: While I agree that there are some clear examples where methanol masers appear to exhibit Keplerian rotation in a disk about a massive protoOB star, in the majority of cases where alignment of masers is seen, at least in the survey of Walsh et al. (1997 and 1998, MNRAS 301, 640), the velocity range amongst the spots is not consistent with Keplerian rotation in a disk, unless particularly massive $\left(M>100 \mathrm{M}_{\odot}\right)$ central objects exist within them. The alignments may be produced in the wrinkled surface of a shock front, perhaps driven by winds from a nearby embedded source.

R.P. Norris: G309.92 and G351.42 (shown in this paper) initially looked like they were too large to sustain Keplerian rotation around a reasonable mass, but higher resolution (VLBI) observations showed quite unambiguously that each consisted of two neighbouring stars. While it is important to keep an open mind at this stage, as there may be more than one class of linear maser, we also have to be very careful about the effects of confusion, as most of these sources are located in rich and complex star formation regions, and high-quality, highresolution data are essential to separate them.

J. Storey: Could you please elaborate on the degree to which these masers are beamed, and how this affects the observational statistics of edge-on versus face-on disks?

R.P. Norris: Because the masers in the plane of the disk can have a greater coherence length than those perpendicular to the disk, maser emission viewed in the plane will be brighter than emission viewed from the pole. So, in a fluxlimited sample of masers, we expect to see proportionally more edge-on disks than face-on disks, which presumably explains why we see so many $(\sim 30 \%)$ edge-on disks.

T. Geballe: How long are the maser-emitting columns in the disks compared to the dimensions of the disk?

R.P. Norris: This is an excellent question to which I do not, unfortunately, have a good answer. Because we have no detailed models of the maser mechanism, we cannot use brightness temperature to derive a gain length. So the masers could be anywhere from $\sim 10^{11}(1 \mathrm{AU})$ to $\sim 10^{14} \mathrm{~m}(1000 \mathrm{AU})$ in length.

Question: In view of the fact that $\mathrm{H}_{2} \mathrm{O}$ masers and class $\mathrm{I} \mathrm{CH}_{3} \mathrm{OH}$ masers trace outflows, is it not likely that some class II $\mathrm{CH}_{3} \mathrm{OH}$ masers may trace outflowing gas rather than the disk or be contaminated by outflowing gas?

R. P. Norris: Certainly. The disks I have shown here represent only about $30 \%$ of class II maser sources. The rest have complex distributions, similar to $\mathrm{OH}$ and $\mathrm{H}_{2} \mathrm{O}$ masers, which we have not been able to model. It is quite possible that they are located in shocks or outflows. 


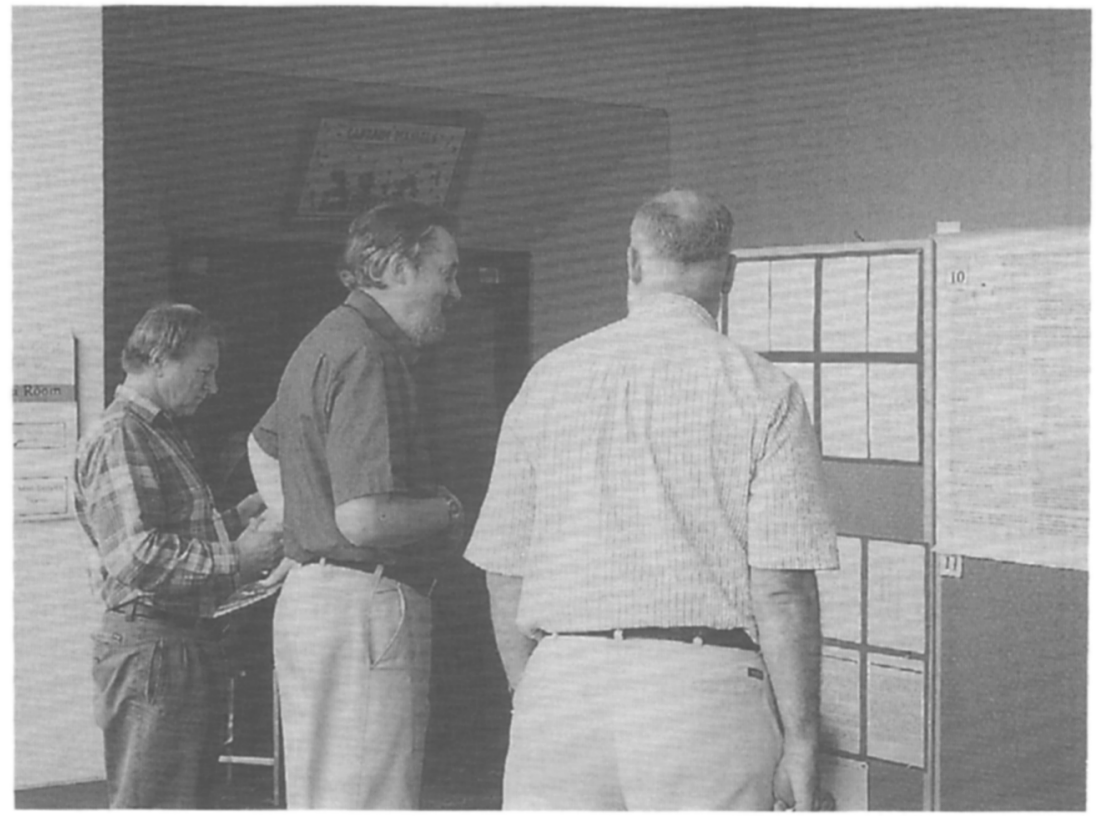

